REVISTA DE ESTUDIOS E INVESTIGACIÓN

EN PSICOLOGÍA Y EDUCACIÓN

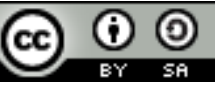

ISSN: 1138-1663; elSSN: 2386-7418

UDC / UMinho

2021, Vol. 8, No. 2, 180-197.

DOI: https://doi.org/10.17979/reipe.2021.8.2.8458

\title{
Evaluación de una intervención preventiva del uso de drogas al agregar un componente para padres
}

\section{Evaluation of a preventive intervention for drug use by adding a component for parents}

\author{
Jorge Alberto Valadez-García (iD) https://orcid.org/0000-0002-0503-0060 \\ Universidad de Guadalajara, Centro Universitario de Ciencias de la Salud: \\ https://www.cucs.udg.mx \\ Guadalajara, Jalisco - México \\ Roberto Oropeza-Tena (iD) https://orcid.org/0000-0002-2561-2164 \\ Universidad Michoacana de San Nicolás de Hidalgo: https://www.umich.mx \\ Morelia, Michoacán - México \\ Guadalupe Vasquez (D) https://orcid.org/0000-0002-8441-0300 \\ Vasquez Clinical Services \\ Clovis, CA - United States
}

\footnotetext{
Para realizar esta investigación el primer autor contó con una beca del Consejo Nacional de Ciencia y Tecnología (CONACYT) de México.

Correspondencia relativa a este artículo: Roberto Oropeza-Tena - roberto.oropeza@umich.mx
} 


\title{
Resumen
}

El objetivo de este estudio fue evaluar la eficacia de la Intervención Preventiva para Estudiantes Adolescentes en Riesgo (IPEA-R) cuando se combina con la Intervención Mínima para Padres de Adolescentes en Riesgo (IMPA-R). El estudio tuvo un diseño cuasi-experimental. La muestra estuvo formada por 25 estudiantes de secundaria, 10 mujeres y 15 varones (edad promedio 12.10 años, $D E=0.32$ ), y se dividió en dos grupos: el grupo IPEA-R $(n=15)$ y el grupo IPEA-R+IMPA-R $(n=10)$. A ambos grupos se les aplicó la IPEA-R, que consta de una sesión se evaluación, ocho de intervención y dos de seguimiento. El segundo grupo recibió además la IMPA-R dirigida a sus padres, que consiste en cuatro sesiones de intervención y dos de seguimiento. Los instrumentos utilizados para el estudio fueron: la Escala de Detección de Consumo de Alcohol, Tabaco y Marihuana, la Escala de Resistencia a la Presión de Amigos, la Escala para la Evaluación del Manejo de Emociones, el Cuestionario de Percepción de Riesgos del Consumo de Drogas, y la Escala de Ambiente Familiar. Se llevó a cabo un ANOVA mixto para identificar diferencias entre los dos grupos en las mediciones pre-test, post-test, primer seguimiento y segundo seguimiento. Se encontraron diferencias estadísticamente significativas en relación con: claridad emocional, reparación emocional, comunicación del hijo, apoyo del hijo, comunicación de los padres, y hostilidad y rechazo. La intervención IPEA-R+IMPA-R mostró efectos positivos en el manejo de emociones y la percepción del ambiente familiar en los adolescentes.

Palabras clave: adolescencia, consumo de drogas, estudiantes de secundaria, intervención cognitivo-conductual, prevención de adicciones

\begin{abstract}
The aim of the study was to evaluate the efficacy of the Preventive Intervention for Student Adolescents at Risk (PISA-R) when combined with the Minimum Intervention for Parents of Adolescents at Risk (MIPA-R). The study had a quasi-experimental design. The sample comprised 25 high school students, 10 female and 15 male (average age 12.10 years, $S D=0.32)$, and was divided into two groups: the PISA-R group $(n=15)$ and the PISA-R+MIPA-R group $(n=10)$. The PISA-R, which consists of an evaluation session, eight intervention sessions and two follow-ups, was applied to both groups. The second group also received the MIPA-R aimed at their parents, which consisted of four intervention sessions and two follow-up sessions. The instruments used for the study were the Detection of Alcohol, Tobacco and Marijuana Scale, the Resistance to Peer Pressure Scale, the Emotion Management Assessment Scale, the Perception of Drug Use Risk Questionnaire, and the Family Environment Scale. A mixed ANOVA was carried out to identify differences between the two groups in the pre-test, post-test, first follow-up and second follow-up measurements. Statistically significant differences were found in relation to emotional clarity, emotional repair, child communication, child support, parent communication, and hostility and rejection. The PISA-R+MIPA-R intervention showed a positive effect on emotion management and perception of family environment among adolescents.
\end{abstract}

Keywords: adolescence, drug use, secondary school students, cognitive-behavioural intervention, addiction prevention 
El consumo de drogas en la etapa de la adolescencia ocasiona diversos daños a la salud que impactan en su desarrollo individual, familiar y social (Jenkins, 2017; Stanis \& Andersen, 2014). Además, el inicio temprano del consumo de sustancias psicoactivas está relacionado con la posible adquisición de una dependencia, debido a la vulnerabilidad biológica, psicológica y social en que se encuentran los adolescentes (United Nations, Office on Drugs and Crime, 2020).

De acuerdo a la Encuesta Nacional de Consumo de Drogas en Estudiantes (ENCODE) realizada en el 2014, se reportó que en México el 6.3\% de los estudiantes de secundaria son fumadores actuales (Villatoro-Velázquez et al., 2015a). Con relación en el consumo de alcohol, la prevalencia en estudiantes de secundaria y bachillerato fue de 53.2\% (Villatoro-Velázquez et al., 2015b). Por último, se reportó que el porcentaje de estudiantes de secundaria y bachillerato que alguna vez consumieron cualquier droga ilegal fue de 17.2\% en total; y se encontró que la marihuana fue la droga de mayor consumo (Villatoro-Velázquez et al., 2015c). Estos datos epidemiológicos indican la importancia de contar con intervenciones preventivas para evitar el uso de drogas en adolescentes escolarizados.

Las intervenciones tempranas dirigidas a adolescentes que inician el consumo de drogas y los tratamientos cognitivo conductuales han mostrado que son efectivos para reducir y eliminar el consumo de sustancias y otras conductas de riesgo (Benningfield et al., 2015; Windsor et al., 2015). Se ha identificado que la combinación del manejo de contingencias, la entrevista motivacional y la terapia cognitivo-conductual son las mejores herramientas para abordar el fenómeno de la adicción a las drogas (García-Caballero et al., 2018; González y López, 2017). Por su lado, las intervenciones preventivas cuentan con componentes que aumentan los factores de protección, se centran en el desarrollo positivo de los jóvenes (Catalano et al., 2004) y desarrollan habilidades socio-emocionales (Benningfield et al., 2015; Botvin et al., 2018).

También se han considerado efectivas las intervenciones preventivas basadas en la familia, las cuales mejoran la calidad de las relaciones y enseñan habilidades para negociar, monitorear, resolver problemas y generar un ambiente familiar saludable (Van y Fosco, 2016). En una revisión sistemática realizada con 42 estudios se indicó que las intervenciones preventivas dirigidas a padres o tutores podrían ser efectivas para prevenir y disminuir el consumo de tabaco, alcohol y drogas ilegales en sus hijos, la mayoría de ellas realizadas en el contexto escolar (Allen et al., 2016).

En un meta-análisis llevado a cabo con nueve estudios donde se evaluaron intervenciones de prevención selectiva del consumo de drogas en adolescentes dirigidas a sus padres, se encontraron tamaños del efecto altos para variables relacionadas con factores de protección 
como las relaciones familiares $(d=0.82)$ y la parentalidad positiva $(d=0.71)$. Con relación en el consumo de sustancias se observó un menor tamaño del efecto $(d=0.21)$ (Valero et al., 2017). Es importante considerar que las conclusiones entre los estudios del meta-análisis no son contundentes debido a que las intervenciones evaluadas mostraron una alta heterogeneidad.

También se han desarrollado intervenciones para adolescentes y sus padres, este es el caso de los programas Familias Fuertes y Keepin'it REAL (Marsiglia et al., 2016; Orte et al., 2019), los cuales paralelamente aplican una intervención para jóvenes y una intervención para padres, su principal objetivo es mejorar las relaciones familiares para prevenir el consumo de drogas en los adolescentes. En el caso de Keepin'it REAL se mostró que trabajar con padres e hijos tuvo un efecto significante en las normas anti-alcohol $(\theta=.170, p<.01)$, así como un efecto en la disminución de la cantidad de cigarros fumados $(\beta=-.100, p<.01)$ a diferencia del grupo en el que solo se intervino con los adolescentes (Marsiglia et al., 2016). En el caso del programa Familias Fuertes el tamaño del efecto de la intervención fue alto $(d=0.61037)$, en comparación con el grupo control (Orte et al., 2019).

En México existen organizaciones dedicadas a la prevención de las adicciones (Centros de Integración Juvenil, Centros de Atención Primaria en Adicciones, Instituto Mexiquense Contra las Adicciones, etc.), no obstante, a falta de un sistema de evaluación de las intervenciones, no se cuenta con suficiente evidencia de la eficacia de muchas de las intervenciones aplicadas en el país (Castro et al., 2015). Lo anterior indica la necesidad de desarrollar intervenciones preventivas para evitar el consumo de drogas en adolescentes, que cuenten con evaluaciones empíricas de los resultados y puedan ser replicables (Botvin et al., 2018).

Para esta investigación se consideró el principio propuesto por el National Institute of Drug Abuse (2020) para las intervenciones preventivas en estudiantes de educación media, las cuales deben considerar aumentar las habilidades sociales relacionadas con la resistencia a la presión para consumir drogas, aumentar la autoeficacia para no consumirlas y aumentar la percepción de riesgo en torno a ellas.

Una propuesta para prevenir el consumo de drogas en estudiantes de nivel secundaria es la Intervención Preventiva para Estudiantes Adolescentes en Riesgo (IPEA-R), la cual es una intervención basada en la evidencia con efectos positivos en la disminución de la frecuencia de consumo de tabaco y alcohol, y que además mejora las habilidades personales enfocadas a evitar el consumo de droga en los adolescentes (Valadez-García y Oropeza-Tena, 2020).

Por todo lo anterior y considerando la importancia de las intervenciones para padres, esta investigación tuvo como objetivo evaluar la eficacia de la IPEA-R agregándole la Intervención Mínima para Padres de Adolescentes en Riesgo (IMPA-R). 
La hipótesis fue que al agregar la IMPA-R los adolescentes tendrían puntuaciones significativamente mayores en las habilidades de resistencia a la presión de amigos, manejo de emociones, percepción de riesgo del consumo de drogas y ambiente familiar, además de mostrar una disminución de la frecuencia del consumo de tabaco, alcohol y marihuana. Para obtener evidencia sobre esto, se realizó un estudio cuasi-experimental con pretest-postest y seguimientos a los dos grupos en estudio.

\section{Método}

\section{Participantes}

Se seleccionó de manera intencional una escuela secundaria privada donde había la posibilidad de acceder a la población en estudio. Este plantel educativo está localizado en Jalisco, México. La escuela tenía dos salones de primero de secundaria. Se trabajó con el total de estudiantes de cada grupo.

Los criterios de inclusión para la IPEA-R fueron: a) alumno regular del primer grado de secundaria, b) participar de manera voluntaria y firmar su consentimiento de participación, c) entregar el consentimiento informado firmado por los padres. Para la IMPA-R, los criterios de inclusión fueron: a) ser padre, madre o tutor de alumno que participa en la IPEA-R, b) participar voluntariamente y firmar su consentimiento informado.

El grupo IPEA-R estuvo formado por 15 estudiantes (5 mujeres y 10 varones) de 12 y 13 años $(M=12.20, D E=0.41)$, mientras que el grupo IPEA-R+IMPA-R lo formaron 10 estudiantes (5 mujeres y 5 varones) del mismo rango de edad $(M=12.10, D E=0.32)$.

Participaron, asistiendo a todas las sesiones, 7 madres y 3 padres de entre 35 y 43 años de edad $(M=38.1, D E=2.12)$, de distintas familias, todas biparentales con hijos biológicos.

A un grupo de estudiantes de secundaria solo se les aplicó la IPEA-R, mientras que al otro grupo de estudiantes se les aplicó la IPEA-R junto con la IMPA-R dirigida a sus padres.

\section{Instrumentos}

Escala de Detección de Consumo de Alcohol, Tabaco y Marihuana (EDCATM) (Tiburcio et al., 2016). Proporciona información sobre el consumo de sustancias a lo largo de la vida, consta de ocho reactivos. Se obtuvo un alfa de Cronbach de .83 para tabaco, .76 para alcohol y .73 para marihuana en población mexicana.

Escala de Resistencia a la Presión de los Amigos para el Consumo de Alcohol (ERPACA) (Sánchez-Xicotencatl et al., 2013). Evalúa la capacidad del adolescente para rechazar el 
consumo de alcohol cuando no quiere hacerlo, aunque los amigos lo presionen para que lo haga. Tiene seis reactivos que explicaron el $47.41 \%$ de la varianza total y obtuvo un alfa de Cronbach de .77 , datos procedentes de México.

\section{Escala para la Evaluación de la Expresión, Manejo y Reconocimiento de Emociones (EEEMRE)}

(Fernández-Berrocal et al., 2004). Evalúa las destrezas para ser conscientes de las propias emociones, así como la capacidad para regularlas. La escala se compone de 24 ítems que se agrupan en las siguientes dimensiones: a) Atención emocional, que se refiere a la capacidad para sentir y expresar las emociones de forma adecuada (alfa de Cronbach $=.90 ; \mathrm{b}$ ) Claridad emocional, referida a la comprensión de los propios estados emocionales $(\alpha=.90)$; y d) Reparación emocional, que evalúa la capacidad percibida para regular los propios estados emocionales de forma correcta $(\alpha=.86)$. Los datos sobre fiabilidad proceden del estudio original realizado en España.

Cuestionario de Percepción de Riesgo hacia el Consumo de Drogas (CPRCD) (Uribe et al., 2011).

Este instrumento evalúa la opinión que tiene un individuo acerca del peligro que implica el consumir drogas. Está compuesto de 21 ítems y validado en población mexicana. La fiabilidad para la escala completa es de $\alpha=0.77$.

Escala de Ambiente Familiar (EAF) (Villatoro et al., 1997). Evalúa la percepción que el adolescente tiene del ambiente en su familia. Consta de 18 ítems. La fiabilidad para la escala total fue de $\alpha=.95$, mientras que para cada una de sus dimensiones los valores fueron: .79 para hostilidad y rechazo, .84 para comunicación de los padres, .88 para comunicación del hijo, .83 para el apoyo de los padres, y .71 para el apoyo de los hijos (datos obtenidos en México).

\section{Procedimiento}

\section{Capacitación de las terapeutas}

Participaron dos psicólogas, una de ellas fue la responsable de la aplicación de las intervenciones, mujer de 27 años de edad y con una maestría en psicología. La otra psicóloga fungió como ayudante, mujer de 24 años de edad y con una licenciatura en psicología. Cada una de ellas recibió una capacitación de 12 horas, donde se explicó la teoría, el objetivo de las intervenciones, su estructura didáctica, los ejercicios y tareas, así como el uso de cada uno de los folletos de trabajo de cada una de las intervenciones. También se les entregó a las psicólogas una lista de cotejo por cada sesión, para que pudieran realizar la intervención de manera sistemática. Los investigadores supervisaron y dieron seguimiento sesión por sesión a las psicólogas con relación en la aplicación de las intervenciones. 


\section{Aplicación de la intervención}

Para cada uno de los grupos se trabajó en sus respectivas aulas. Cada semana las psicólogas trabajaban primero con los estudiantes del grupo IPEA-R y después con el IPEAR+IMPA-R. Y una vez cada dos semanas en el aula audiovisual se trabajó con el grupo de padres del IPEA-R+IMPA-R. La intervención se realizó en un periodo de dos meses, sin considerar los seguimientos. La directora de la secundaria determinó a que grupo se aplicaría cada intervención, considerando elegir para aplicar la IMPA-R al grupo con padres más participativos, según su experiencia.

\section{Intervenciones}

A continuación se explica a grandes rasgos la estructura de las intervenciones IPEA-R y IMPA-R, número de sesiones para cada intervención, frecuencia y duración, así como los objetivos a lograr. El diseño de las intervenciones se hizo por medio de un procedimiento sistematizado de revisión de la literatura, evaluación por jueces expertos en el tema y pilotajes previos a la aplicación de las intervenciones (Valadez et al., 2020).

Intervención Preventiva para Estudiantes Adolescentes en Riesgo (IPEA-R). Esta intervención está dirigida a adolescentes que inician la secundaria, se aplica en el contexto escolar y fue diseñada con base en una revisión de la literatura científica sobre intervenciones preventivas que fueron evaluadas empíricamente (Allen et al., 2016; Catalano et al., 2004; Stanis y Andersen, 2014). Se incluyeron los estándares internacionales para intervenciones preventivas con resultados eficaces propuestos por la UNODC (2015): a) mejorar en los jóvenes sus habilidades personales y sociales para evitar el consumo de drogas b) impartir la intervención a través de una serie de sesiones estructuradas y con seguimientos al terminar la intervención, c) sesiones principalmente interactivas y d) impartido por profesionales capacitados. Y además se consideraron las sugerencias obtenidas en un estudio exploratorio realizado a profesionales expertos en prevención de las adicciones de la región centro occidente de México (Valadez et al., 2018), en el que brindan sugerencias de los tópicos que debe contener una intervención preventiva eficaz. Se compone de una sesión de evaluación, ocho sesiones de intervención y dos de seguimiento (al mes y a los tres meses de haber finalizado la intervención). Se aplica una sesión por semana con duración de 50 minutos.

En la sesión de evaluación se aplicaron los instrumentos para realizar la medición pretest y se realizó una inducción a la IPEA-R. Los objetivos de la intervención fueron los siguientes: 1) Enseñar a las consecuencias del consumo de drogas, 2) Analizar las consecuencias de sus conductas saludables versus conductas de riesgo, 3) Entrenar en estrategias de auto-control, 4) 
Identificar pensamientos irracionales y transformarlos en pensamientos funcionales, 5) Enseñar estrategias de afrontamiento para el enojo, la tristeza y la euforia, 7) Reconocer y resistir las presiones sociales para usar drogas.

Al finalizar la última sesión de la IPEA-R se aplicaron los instrumentos para la medición postest. Después se realizaron los seguimientos al mes y a los tres meses de haber finalizado la intervención. En estas sesiones de seguimiento los objetivos fueron revisar el logro de la meta de no consumo, diseñar planes de acción para posibles obstáculos y aplicar los instrumentos de medición.

Intervención Mínima para Padres de Adolescentes en Riesgo (IMPA-R). Es una intervención preventiva dirigida a padres o tutores de adolescentes escolarizados y fue diseñada con los mismos fundamentos que la IPEA-R, referidos anteriormente. La IMPA-R se compone de cuatro sesiones de intervención y dos sesiones de seguimiento. Se aplica una sesión cada dos semanas con duración de 60 minutos, y paralelamente a la IPEA-R. Los objetivos de la intervención fueron los siguientes: a) Entrenar a los padres en estrategias para mejorar la comunicación familiar, b) entrenar a los padres en estrategias para aumentar las conductas saludables y disminuir conductas de riesgo, c) establecer reglas claras y lograr un adecuado monitoreo del adolescente.

Después de un mes de la última sesión se realizó el primer seguimiento y tres meses después el segundo seguimiento. En estas sesiones los objetivos fueron analizar obstáculos y realizar planes de acción para que los padres continúen aplicando las estrategias aprendidas.

\section{Consideraciones éticas}

De acuerdo con los preceptos del Código Ético del Psicólogo publicado por la Sociedad Mexicana de Psicología (2010), se identificó que esta investigación tiene riesgos mínimos para los participantes. Se presentó el proyecto de investigación a la directora de la escuela secundaria y, una vez aceptado, se programaron las fechas de intervención. Cada estudiante aceptó participar voluntariamente en la investigación y sus padres (o tutores) otorgaron el consentimiento informado. Este consentimiento se basó en la Ley General de Salud (2015) de los Estados Unidos Mexicanos, Capítulo 5o "Investigación para la salud", Artículo 100.

\section{Análisis de datos}

Se realizó un análisis de varianza factorial mixto para identificar si existían diferencias estadísticamente significativas entre el grupo 1 (IPEA-R) y el grupo 2 (IPEA-R+IMPA-R), en los resultados de las mediciones pretest, postest, primer seguimiento y segundo seguimiento, de 
las variables consumo de drogas, resistencia a la presión, manejo de emociones, percepción de riesgo asociados al consumo de drogas y ambiente familiar. Para identificar el tamaño del efecto del ANOVA mixto se realizaron pruebas del valor eta cuadrado parcial $(\eta 2 p)$. Los valores de referencia para el tamaño del efecto del $\eta 2 p$ fueron: pequeño $=.01 ;$ mediano $=.06 ;$ y grande $=.14$ (Castillo-Blanco y Alegre, 2015). Se realizó el análisis estadístico con el programa IBM SPSS v. 21 para Windows.

\section{Resultados}

Es importante resaltar que, en cuanto a las frecuencias del consumo de alcohol en los últimos 30 días, se observó que en el Grupo 1 (IPEA-R) y en el Grupo 2 (IPEA-R+IMPA-R) hubo una disminución del consumo de bebidas alcohólicas reportado durante la intervención: de tres y dos bebidas respectivamente en el pretest, a cero bebidas en el segundo seguimiento en ambos grupos.

Durante la investigación, no se reportó ningún consumo de tabaco ni de marihuana al momento de las evaluaciones en ninguno de los grupos en estudio.

En la Tabla 1 se muestran los promedios de la evaluación pretest y segundo seguimiento para todas las variables dependientes, como se puede observar, todas las variables tuvieron una media mayor en la evaluación del segundo seguimiento en comparación con la evaluación pretest, con excepción de la variable hostilidad y rechazo de la EAF, que se interpreta de manera inversa, es decir, menor puntaje refleja menor hostilidad y rechazo.

También, en la Tabla 1 se pueden identificar las variables dependientes que mostraron una diferencia estadísticamente significativa en el análisis inter-sujetos entre el grupo 1 y el grupo 2 en las mediciones pretest, postest, primer seguimiento y segundo seguimiento. El grupo 2 obtuvo mejores puntajes en claridad emocional, reparación emocional de la EEEMRE, comunicación del hijo, apoyo del hijo, comunicación de los padres y hostilidad-rechazo de la EAF, en comparación con el grupo IPEA-R. El tamaño del efecto obtenido por el valor de eta cuadrado parcial mostró un tamaño del efecto grande.

Finalmente, es importante mencionar que las variables dependientes que no mostraron una diferencia estadísticamente significativa en el análisis inter-sujetos entre el grupo 1 y el grupo 2 en las mediciones pretest-postest- primer seguimiento- segundo seguimiento fueron: resistencia a la presión de amigos, atención emocional del EEEMRE, percepción de riesgo del consumo de tabaco, alcohol y marihuana, así como la percepción que el adolescente tiene del apoyo de los padres del EAF. 


\section{Tabla 1}

Prueba ANOVA de efectos inter-sujetos de todas las variables en estudio

\begin{tabular}{|c|c|c|c|c|c|}
\hline \multirow[b]{2}{*}{ Variables } & \multirow[b]{2}{*}{ Grupo } & Pretest & 2do Segto. & \multirow[b]{2}{*}{$F$} & \multirow[b]{2}{*}{$\eta^{2} p$} \\
\hline & & $M(D E)$ & $M(D E)$ & & \\
\hline \multirow{2}{*}{$\begin{array}{l}\text { Resistencia a la presión de amigos } \\
\text { (ERPACA) }\end{array}$} & 1 & $21.53(3.09)$ & $23.53(0.99)$ & \multirow[t]{2}{*}{.88} & \multirow[t]{2}{*}{.037} \\
\hline & 2 & $21.90(1.66)$ & $24.00(0.00)$ & & \\
\hline \multicolumn{6}{|l|}{ Manejo de emociones (EEEMRE) } \\
\hline \multirow[t]{2}{*}{ Atención emocional } & 1 & $25.93(7.52)$ & $28.13(5.19)$ & \multirow[t]{2}{*}{.10} & \multirow[t]{2}{*}{.004} \\
\hline & 2 & $24.70(7.83)$ & $30.20(4.05)$ & & \\
\hline \multirow[t]{2}{*}{ Claridad emocional } & 1 & $22.67(5.39)$ & $28.87(3.06)$ & \multirow[t]{2}{*}{$20.06 * * *$} & \multirow[t]{2}{*}{.466} \\
\hline & 2 & $29.10(6.83)$ & $36.80(3.61)$ & & \\
\hline \multirow[t]{2}{*}{ Reparación emocional } & 1 & $26.73(7.50)$ & 33.33 (3.59) & \multirow[t]{2}{*}{$12.86^{* *}$} & \multirow[t]{2}{*}{.359} \\
\hline & 2 & $31.30(6.14)$ & $39.10(1.19)$ & & \\
\hline \multicolumn{6}{|l|}{ Percepción de riesgo sobre (CPRCD) } \\
\hline \multirow[t]{2}{*}{ Tabaco } & 1 & $6.13(1.12)$ & $8.00(0.00)$ & \multirow[t]{2}{*}{.23} & \multirow[t]{2}{*}{.010} \\
\hline & 2 & $6.40(0.96)$ & $8.00(0.00)$ & & \\
\hline \multirow[t]{2}{*}{ Alcohol } & 1 & $8.60(2.13)$ & $11.13(0.99)$ & \multirow[t]{2}{*}{.78} & \multirow[t]{2}{*}{.033} \\
\hline & 2 & $9.00(1.56)$ & $11.40(0.96)$ & & \\
\hline \multirow[t]{2}{*}{ Marihuana } & 1 & $5.80(1.20)$ & $7.20(0.86)$ & \multirow[t]{2}{*}{.13} & \multirow[t]{2}{*}{.005} \\
\hline & 2 & $5.40(0.96)$ & $7.70(0.48)$ & & \\
\hline \multicolumn{6}{|l|}{ Ambiente familiar (EAF) } \\
\hline \multirow[t]{2}{*}{ Comunicación del/a hijo/la } & 1 & $7.07(2.81)$ & $7.80(1.85)$ & \multirow[t]{2}{*}{$5.92^{*}$} & \multirow[t]{2}{*}{.205} \\
\hline & 2 & $8.00(3.23)$ & $10.20(1.75)$ & & \\
\hline \multirow[t]{2}{*}{ Apoyo del/la hijo/a } & 1 & $8.13(2.23)$ & $10.07(1.22)$ & $7.07^{*}$ & .235 \\
\hline & 2 & $9.40(2.71)$ & $11.20(0.91)$ & & \\
\hline Comunicación de padres & 1 & $7.27(2.18)$ & $9.80(1.56)$ & $8.11^{* *}$ & .261 \\
\hline & 2 & $8.40(2.01)$ & $11.50(0.52)$ & & \\
\hline Apoyo de padres & 1 & $8.87(2.32)$ & $10.60(1.24)$ & 2.44 & .096 \\
\hline & 2 & $9.50(2.83)$ & $11.70(0.67)$ & & \\
\hline Hostilidad-rechazo & 1 & $9.93(2.96)$ & $8.80(1.78)$ & $14.13^{* * *}$ & 381 \\
\hline & 2 & $8.60(1.57)$ & $6.40(0.51)$ & & \\
\hline
\end{tabular}

Nota. Grupo: 1 = IPEA-R, 2 = IPEA-R + IMPA-R

$* p<.05 ; * * p<.01 ; * * * p<.001$

\section{Discusión}

Esta investigación sugiere que al combinar la IPEA-R + IMPA-R se obtienen mejores resultados en el manejo de emociones y la percepción del ambiente familiar de los estudiantes adolescentes en comparación a solamente intervenir con la IPEA-R.

En cuanto al consumo de bebidas alcohólicas, ambos grupos en estudio pasaron de tres (Grupo 1) y dos bebidas estándar (Grupo 2) en el pretest a cero bebidas en el segundo seguimiento. En otra investigación donde se evaluó la eficacia de la IPEA-R, se observó que en el grupo a quienes se le aplicó la intervención preventiva se logró una disminución de nueve 
bebidas alcohólicas en el pretest a tres bebidas en el segundo seguimiento, mientras que en el grupo control se observó un aumento de seis bebidas alcohólicas en el pretest a 16 bebidas en el segundo seguimiento (Valadez-García y Oropeza-Tena, 2020). En otras investigaciones también se reportó disminución del consumo de bebidas alcohólicas en los jóvenes como resultado de intervenciones preventivas conjuntas con sus padres (Marsiglia et al., 2016; Orte et al., 2019)

De acuerdo a la ENCODE, en México la prevalencia del consumo de alcohol en estudiantes de 12 años de edad o menos fue de $4 \%$, en estudiantes de 17 años de edad de $28.8 \%$ y en estudiantes de 18 años o más fue de $37.7 \%$, lo cual nos india un aumento significativo en la mediad en que los jóvenes van creciendo (ENCODE, 2015b). Por lo anterior, se puede decir que la IPEA-R por sí misma y junto con la IMPA-R pueden ser una alternativa para disminuir la frecuencia del consumo de bebidas alcohólicas en adolescentes escolarizados.

Por otro lado, la combinación de la IPEA-R + IMPA-R mejoró los resultados en torno a la claridad emocional y la reparación emocional, estos resultados coinciden con la investigación donde se evaluó la IPEA-R en comparación con un grupo control (Valadez-García y OropezaTena, 2020); en contraste, en otro estudio se encontró que los jóvenes con bajos niveles de reparación emocional tuvieron mayor consumo de marihuana (González-Yubero et al., 2020).

Es importante mencionar que la IPEA-R contiene dos sesiones dedicadas al manejo de emociones y que en la IMPA-R se enseña a los padres algunas habilidades para comunicarse y motivar a sus hijos eficazmente, factores que posiblemente repercutan en los adolescentes y su capacidad de comprender y regular sus emociones (Alonso-Castillo et al., 2017), se ha demostrado que las intervenciones centradas en las emociones favorecen el desarrollos de habilidades sociales, autocontrol y empatía (Merchán et al., 2014).

En la comparación del ambiente familiar entre los dos grupos, se observó que el grupo 2 obtuvo mejores puntuaciones en la escala EAF, es decir, los jóvenes evaluados perciben tener buena comunicación con sus padres y no sienten hostilidad; por otro lado, creen que no reciben el suficiente apoyo por parte de los padres. Es probable que la menor percepción de los jóvenes sobre el apoyo de los padres hacia ellos se deba al egocentrismo característico en el desarrollo del adolescente (Mandavia et al., 2016).

El clima familiar negativo (estrés, rechazo), conflicto familiar excesivo (hostilidad, protección excesiva) y falta de comunicación son las variables del ámbito familiar que más frecuentemente se relacionan con el consumo de drogas por parte de un miembro de la familia (Alonso-Castillo et al., 2017; Valero et al., 2017; Van y Fosco, 2016). Por lo tanto, contar con 
intervenciones que mejoren el ambiente familiar podría contribuir a prevenir el consumo de drogas.

Respecto a las variables resistencia a la presión de amigos, atención emocional, percepción de riesgo del consumo de drogas y apoyo de los padres no se encontraron diferencias estadísticamente significativas entre los grupos en estudio. Por otro lado, es importante mencionar que en la comparación de las medias entre la medición pretest y el segundo seguimiento, ambos grupos en estudio obtuvieron mejores puntajes en todas estas variables. Lo anterior nos puede indicar que para estas variables la IPEA-R tiene un efecto positivo ya que puede favorecer a mejorar los puntajes (Valadez-García y Oropeza-Tena, 2020), pero al agregar la IMPA-R no se muestra una diferencia significativa.

A pesar de que en esta investigación no se encontró una diferencia significativa en el incremento de la habilidad de resistencia a la presión, es importante mencionar que se puede enseñar a estudiantes adolescente que no ha consumido drogas, como un recurso preventivo (Schinke et al., 2016), ya que se ha demostrado la influencia que pueden tener los amigos respecto a tener una actitud favorable hacia el consumo de drogas, lo cual constituye un factor de riesgo que incrementa la vulnerabilidad de los menores (Platas-Ferreiro, 2015). Por otro lado, el aumento de la percepción de riesgo implica considerar y evaluar la información que se tiene sobre los daños del consumo de drogas y las experiencias personales (Tan et al., 2018). La IPEA$\mathrm{R}$ contiene componentes para mejorar las habilidades de resistencia a la presión y el aumento de la percepción de riesgo del consumo de drogas y en la IMPA-R se enseña a los padres a tener un monitoreo adecuado de los hijos.

Otras intervenciones que sincronizan el trabajo con jóvenes y sus padres y que han mostrado resultados positivos es el de Fortalecimiento Familiar (Orte et al., 2019) y Keepin'it REAL (Marsiglia et al., 2016), los cuales abarcan aspectos como la comunicación, la cohesión y la organización. Sin embargo, se encontraron dificultades en el momento de tratar de replicar los resultados de la intervención de Fortalecimiento Familiar (Gorman, 2017; Kumpfer et al., 2018), este es el riesgo de las intervenciones adaptada de otros países (Bröning et al., 2017).

Finalmente es importante mencionar que tanto la IPEA-R como la IMPA- $R$ son intervenciones que de manera dinámica y a través de ensayos conductuales y técnicas cognitivas favorecen a los adolescentes y sus padres en el desarrollo de habilidades para prevenir el consumo de drogas.

Como conclusión y con base en los resultados obtenidos en esta investigación, se puede decir que la IPEA-R es una intervención preventiva eficaz para mejorar algunas habilidades 
relacionadas con la prevención del inicio del consumo de drogas en estudiantes de secundaria y al agregar el componente para padres, por medio de la IMPA-R, se mejoran las variables emocionales (claridad y reparación emocional) y la percepción del ambiente familiar en los adolescentes (comunicación del hijo, apoyo del hijo, comunicación de padres y hostilidadrechazo), en comparación a los estudiantes que solo se les aplica la IPEA-R.

Una de las limitaciones de esta investigación es la correspondiente al tamaño de la muestra y al tipo de muestreo; además tuvo un sesgo muestral, debido a que el estudio se realizó solamente en una escuela privada y la asignación del grupo experimental 2 fue determinado por la directora, quien, según su experiencia, seleccionó al grupo con padres más participativos. Otra limitante que se debe considerar es la deseabilidad social y el efecto que puede tener el contestar varias veces, en poco tiempo, los instrumentos de evaluación.

Para próximas investigaciones se sugiere incluir un muestreo probabilístico, una muestra más representativa y contar con seguimientos a largo plazo. También se sugiere agregar, además de los dos grupos experimentales, un tercer grupo control con adolescentes a quienes no se les aplique ninguna intervención y permanezcan en lista de espera hasta finalizar todas las evaluaciones.

\section{Conflicto de interés}

Los autores de este trabajo declaran que no existe conflicto de intereses.

\section{Agradecimientos}

A las autoridades de la escuela secundaria donde se realizó esta investigación y al Consejo Nacional de Ciencia y Tecnología (CONACYT) por el apoyo económico otorgado, como parte de su programa de becas para estudios de Doctorado.

\section{Referencias}

ALLEN, Michel L.; GARCIA-HUIDOBRO, Diego; PORTA, Carolyn; CURRAN, Dorothy; PATEL, Roman; MILLER, Jonathan; \& BOROWSKY, Iris (2016). Effective parenting interventions to reduce youth substance use: A systematic review. Pediatrics, 138(2), e20154425. https://doi.org/ $10.1542 /$ peds.2015-4425

ALONSO-CASTILLO, María Magdalena; YAÑEZ-LOZANO, Ángeles; \& ARMENDÁRIZ-GARCÍA, Nora Angélica (2017). Funcionalidad familiar y consumo de alcohol en adolescentes de secundaria. Health and Addictions, 17(1), 87-96. https://ojs.haaj.org/?journal=haaj\&page $=$ article\&op=view\&path\%5B\%5D=286 
BENNINGFIELD, Margaret M.; RIGGS, Paula; \& STEPHAN, Sharon Hoover (2015). The role of schools in substance use prevention and intervention. Child and Adolescent Psychiatric Clinics, 24(2), 291-303. https://doi.org/10.1016/j.chc.2014.12.004

BOTVIN, Gilbert J.; GRIFFIN, Kenneth W.; BOTVIN, Caroline; MURPHY, Madhuvanti; \& ACEVEDO, Bianca (2018). Increasing implementation fidelity for school-based drug abuse prevention: effectiveness of enhanced training and technical assistance. Journal of the Society for Social Work and Research, 9(4), 599-613. https://doi.org/10.1086/700972

BRÖNING, Sonja; BALDUS, Christiane; THOMSEN, Monika; SACK, Peter-Michael; ARNAUD, Nicoals; \& THOMASIUS, Rainer (2017). Children with elevated psychosocial risk load benefit most from a family-based preventive intervention: exploratory differential analyses from the German "Strengthening Families Program 10-14" adaptation trial. Prevention Science, 18, 932-942. https://doi.org/10.1007/s11121-017-0797-x

CASTILLO-BLANCO, Ronald; \& ALEGRE, Alberto (2015). Importancia del tamaño del efecto en el análisis de datos de investigación en psicología. Persona, 18, 137-148. https://doi.org/ 10.26439/persona2015.n018.503

CASTRO, María; LLANES, Jorge; AMADOR, Nancy; VILLATORO, Jorge; \& MEDINA-MORA, María Elena (2015). La Prevención del Consumo de Drogas en México. En A. Pérez, J. Mejía \& E. Becoña (Eds). De la prevención y otras historias: historia y evolución de la prevención del consumo de alcohol y drogas en América Latina y en Europa (pp. 243-282). California-Edit.

CATALANO, Richard F.; BERGLUND, M. Lisa; Ryan, Jeanne; LONCZAK, Heather S.; \& HAWKINS, J. David (2004). Positive youth development in the United States: Research findings on evaluations of positive youth development programs. The Annals of the American Academy of Political and Social Science, 591(1), 98-124. https://doi.org/10.1037/1522-3736.5.1.515a FERNÁNDEZ-BERROCAL, Pablo; EXTREMERA, Natalio; \& RAMOS, Natalia (2004). Validity and reliability of the Spanish modified version of the Trait Meta-Mood Scale. Psychological Reports, 94, 751-755. https://doi.org/10.2466/pr0.94.3.751-755

GARCIA-CABALlERO, Anna; TORRENS-LLUCH, Marina; RAMíREZ-GENDRAU, Isabel; GARRIDO, Gemma; VALLÈS, Vicenç; \& ARAGAY, Núria (2018). Eficacia de la intervención motivacional y la terapia cognitivo-conductual para el tratamiento del juego patológico. Adicciones, 30 (3), 219-224. https://www.adicciones.es/index.php/adicciones/article/view/965

GONZÁLEZ, Claudia; \& LÓPEZ, Ana (2017). Revisión del tratamiento psicológico de la adicción al cannabis. Health and Addictions, 17(1), 15-26. https://ojs.haaj.org/?journal=haaj\&page= article\&op=view\&path\%5B\%5D=270 
GONZÁLEZ YUBERO, Sara; PALOMERA MARTÍN, Raquel; LÁZARO-VISA, Susana. Inteligencia emocional rasgo y habilidad como factores asociados al consumo de cannabis en la adolescencia. Adicciones, 33(4), 345-358. https://doi.org/10.20882/adicciones.1364

GORMAN, Dennis (2017). The decline effect in evaluations of the impact of the Strengthening Families Program for Youth 10-14 (SFP 10-14) on adolescent substance use. Children and Youth Services Review, 81, 29-39. https://doi.org/10.1016/j.childyouth.2017.07.009

JENKINS, Emily K.; SLEMON, Allie; \& HAINES-SAAH, Rebecca J. (2017). Developing harm reduction in the context of youth substance use: insights from a multi-site qualitative analysis of young people's harm minimization strategies. Harm Reduction Journal 14(53), 1-11. https://doi.org/10.1186/s12954-017-0180-z

KUMPFER, Karol; SCHEIER, Lawrence; \& BROWN, Jaynie (2020). Strategies to avoid replication failure with evidence-based prevention interventions: case examples from the Strengthening Families Program. Evaluation \& the Health Professions, 43(2), 75-89. https://doi.org/10.1177/0163278718772886

Ley General de Salud (2015). Cámara de diputados del H. Congreso de la Unión. Secretaría General. Secretaría de Servicios Parlamentarios. Estados Unidos Mexicanos. Disponible en http://www.diputados.gob.mx/LeyesBiblio/ref/lgs.htm

MANDAVIA, Amar; ROBINSON, Gabriella G. N.; BRADLEY, Bekh; RESSLER, Kerry J.; \& POWERS, Abigail (2016). Exposure to childhood abuse and later substance use: Indirect effects of emotion dysregulation and exposure to trauma. Journal of Traumatic Stress, 29(5), 422429. https://doi.org/10.1002/jts.22131

MARSIGLIA, Flavio F.; AYERS, Stephanie L.; BALDWIN-WHITE, Adrienne; \& BOOTH, Jaime (2016). Changing Latino adolescents' substance use norms and behaviors: The effects of synchronized youth and parent drug use prevention interventions. Prevention Science, 17(1), 1-12. https://doi.org/10.1007/s11121-015-0574-7

MERCHÁN, Isabel María; BERMEJO, María Luisa; \& GONZÁLEZ, Juan de Dios (2014). Eficacia de un programa de educación emocional en educación primaria. Revista de Estudios e Investigación en Psicología y Educación, 1(1), 91-99. https://doi.org/10.17979/reipe. 2014.1.1.30

National Institute of Drug Abuse (2020). Cómo prevenir el uso de drogas en niños y adolescentes ( $2^{\underline{a}}$ ed.). National Institute of Health. https://www.drugabuse.gov/es/publicaciones/ como-prevenir-el-uso-de-drogas/los-principios-de-la-prevencion 
ORTE, Carmen; BALLESTER, Lluis; AMER, Joan; \& VIVES, Marga (2019). Building family resilience through an evidence-based program: Results from the Spanish Strengthening Families Program. The Family Journal: Counseling and Therapy for Couples and Families, 27(4), 409-417. https://doi.org/10.1177/1066480719872753

PLATAS-FERREIRO, María Lidia (2015). Coetáneos y actitud ante el consumo de drogas en menores. Revista de Estudios e Investigación en Psicología y Educación, Extr.(2), 039-041. https://doi.org/10.17979/reipe.2015.0.02.427

SÁNCHEZ-XICOTENCATL, Omar; ANDRADE, Patricia; BETANCOURT, Diana; \& VITAL, Guadalupe (2013). Escala de resistencia a la presión de los amigos para el consumo de alcohol. Acta de Investigación Psicológica, 3(1), 917-929. https://www.elsevier.es/es-revista-actainvestigacion-psicologica-psychological-111-articulo-escala-resistencia-presion-amigosel-S2007471913709426

SCHINKE, Steven; SCHWINN, Traci; HOPKINS, Jessica; \& WAHLSTROM, Lindsey (2016). Drug abuse risk and protective factors among Hispanic adolescents. Preventive Medicine Reports, 3, 185-188. https://doi.org/10.1016/j.pmedr.2016.01.012

Sociedad Mexicana de Psicología (2010). Código Ético del Psicólogo. Trillas.

STANIS, Jessica J.; \& ANDERSEN, Susan L. (2014). Reducing substance use during adolescence: a translational framework for prevention. Psychopharmacology, 231(8), 1437-1453. https://doi.org/10.1007/s00213-013-3393-1

TAN, Huey Jing; YEE, Anne; SULAIMAN, Ahmad Hatim; AYU, Said; DANAEE, Mahmoud; \& LUA, Ahai (2018). Effects of a school-based substance use prevention program on students in Malaysia. Journal of Health and Translational Medicine, 21(1). 40-46.

TIBURCIO, Marcela; ROSETE-MOHEDANO, Ma. Guadalupe; NATERA, Guillermina; MARTÍNEZ, Nora Angelica; CARREÑO, Silvia; \& PÉREZ, Daniel (2016). Validez y confiabilidad de la prueba de detección de consumo de alcohol, tabaco y sustancias (ASSIST) en estudiantes universitarios. Adicciones, 28(1), 19-27. https://doi.org/10.20882/adicciones.786

United Nations, Office on Drugs and Crime (2020). UNODC/WHO International standards on drug use prevention (Second updated edition). United Nations Publication. https://www.unodc.org/unodc/en/prevention/prevention-standards.html

URIBE-ALVARADO, Isaac; VERDUGO-LUCERO, Julio César; \& ZACARÍAS-SALINAS, Ximena (2011). Relación entre percepción de riesgo y consumo de drogas en estudiantes de bachillerato. Psicología y Salud, 21(1), 47- 55. https://psicologiaysalud.uv.mx/index.php/psicysalud/ article/view/586 
VALADEZ-GARCÍA, Jorge Alberto; \& OROPEZA-TENA, Roberto (2020). Evaluación de la Intervención Preventiva para Estudiantes Adolescentes en Riesgo (IPEA-R) en estudiantes de secundaria. Health and Addictions, 20(2), 157-169. https://doi.org/10.21134/haaj. v20i2.550

VALADEZ-GARCÍA, Jorge Alberto; OROPEZA-TENA, Roberto; SALAZAR-GARZA, Martha Leticia; \& FULGENCIO-JUÁREZ, Mónica (2020). Desarrollo de intervenciones para prevenir el consumo de drogas en estudiantes adolescentes. En María Gabriela, Luna; \& Roberto, Montes (Comp.). Investigación Interinstitucional en Psicología. Nuevos desafíos para el siglo XXI (pp. 91-110). Grañén Porrúa.

VALADEZ, Jorge Alberto; OROPEZA, Roberto; SALAZAR, Martha Leticia; \& MARTínEZ, Kalina (2018). La voz de los profesionales: componentes y sugerencias para los programas de prevención en adicciones. Revista Electrónica de Psicología Iztacala, 21(3), 796-816. https://www.medigraphic.com/cgi-bin/new/resumen.cgi?IDARTICULO=83158

VALERO, María; BALLESTER, Lluís; ORTE, María; \& AMER, Joan (2017). Meta-analysis of familybased selective prevention programs for drug consumption in adolescence. Psicothema, 29(3), 299-305. https://doi.org/10.7334/psicothema2016.275

VAN, Mark; \& FOSCO, Gregory (2016). Family-based approaches to prevention. In Mark J. Van, K.L. Kumpfer, G.M., Fosco, \& M.T. Greenberg (Eds.), Family-based prevention programs for children and adolescents: theory, research, and large-scale dissemination (pp. 1-20). Psychology Press. https://doi.org/10.4324/9781315764917

VILLATORO, Jorge; ANDRADE, Patricia; FLEIZ, Clara; MEDINA-MORA, María Elena; REYES, Isabel; \& RIVERA, Edith (1997). La relación padres-hijos: una escala para evaluar el ambiente familiar de los adolescentes. Salud Mental. 2, (20), 21-27. http://www.revistasalud mental.mx/index.php/salud_mental/article/view/647

VILLATORO-VELÁZQUEZ Jorge; BUSTOS-GAMIÑO, Marycarmen; OLIVA-ROBLES, Natania; FREGOSO-ITO, Diana; MUJICA-SALAZAR, Ailema; MARTÍN DEL CAMPO-SÁNCHEZ, Raul; NANNI-ALVARADO, Ricardo; \& MEDINA-MORA, María Elena (2015a). Encuesta Nacional de Consumo de Drogas en Estudiantes 2014: Reporte de Tabaco. Instituto Nacional de Psiquiatría Ramón de la Fuente Muñiz; Comisión Nacional Contra las Adicciones, Secretaría de Salud. Disponible en: https://www.gob.mx/salud\%7Cconadic/documentos/ encuesta-nacional-de-consumo-de-drogas-en-estudiantes-2014-encode

VILLATORO-VELÁZQUEZ Jorge; BUSTOS-GAMIÑO, Marycarmen; OLIVA-ROBLES, Natania; FREGOSO-ITO, Diana; MUIICA-SALAZAR, Ailema; MARTÍN DEL CAMPO-SÁNCHEZ, Raul; 
NANNI-ALVARADO, Ricardo; \& MEDINA-MORA, María Elena (2015b). Encuesta Nacional de Consumo de Drogas en Estudiantes 2014: Reporte de Alcohol. Instituto Nacional de Psiquiatría Ramón de la Fuente Muñiz; Comisión Nacional Contra las Adicciones, Secretaría de Salud. Disponible en: https://www.gob.mx/salud\%7Cconadic/documentos/ encuesta-nacional-de-consumo-de-drogas-en-estudiantes-2014-encode

VILLATORO-VELÁZQUEZ Jorge; BUSTOS-GAMIÑO, Marycarmen; OLIVA-ROBLES, Natania; FREGOSO-ITO, Diana; MUJICA-SALAZAR, Ailema; MARTíN DEL CAMPO-SÁNCHEZ, Raul; NANNI-ALVARADO, Ricardo; \& MEDINA-MORA, María Elena (2015c). Encuesta Nacional de Consumo de Drogas en Estudiantes 2014: Reporte de Drogas. Instituto Nacional de Psiquiatría Ramón de la Fuente Muñiz; Comisión Nacional Contra las Adicciones, Secretaría de Salud. Disponible en: https://www.gob.mx/salud\%7Cconadic/documentos/ encuesta-nacional-de-consumo-de-drogas-en-estudiantes-2014-encode

WINDSOR, Liliane C.; JEMAL, Alexis; \& ALESSI, Edward J. (2015). Cognitive behavioral therapy: A meta-analysis of race and substance use outcomes. Cultural Diversity and Ethnic Minority Psychology, 21(2), 300-313. https://doi.org/10.1037/a0037929

Fecha de recepción: 19 de mayo de 2021. Fecha de revisión: 1 de septiembre de 2021. Fecha de aceptación: 18 de septiembre de 2021. Fecha de publicación: 1 de diciembre de 2021. 